\title{
The influence of stimulus uncertainty and experimental instructions on visual selection
}

\author{
TERRY T. FAW, ${ }^{2}$ JUM C. NUNNALLY, AND NANCY A. ATOR \\ VANDERBILT UNIVERSITY
}

The looking behavior of adults and children was recorded while they viewed pairs of stimuli differing in stimulus uncertainty. Ss in one experimental group were encouraged to identify the stimuli and indicate whether they had been seen in a previous phase of the experiment. For the remaining Ss, instructions minimized the importance of the stimuli to the experiment. Results indicate that, when stimulus uncertainty was influential, the relationship between stimulus uncertainty and looking time was monotonically increasing. However, stimulus uncertainty was an effective determinant of looking behavior only when Ss were motivated to identify the stimuli. The behavior of adults and children did not differ.

A host of experiments have been designed to examine the influence of stimulus complexity on looking behavior (e.g., Berlyne, 1958; Cantor, Cantor, \& Ditrichs, 1963; Faw \& Nunnally, 1967, 1968; Leckart \& Bakan, 1965). The concept of stimulus complexity has a broad connotation and in those experiments numerous definitions of complexity have been employed (e.g., the number of elements in a display or the number of sides of a random polygon). The present study concerns the relationship between looking behavior and one form of complexity-stimulus uncertainty. Here the term uncertainty is used in the traditional information theoretic sense discussed by Garner (1962, pp. 2-7). Although the results from the majority of those earlier experiments are in keeping with the hypothesis that more complex stimuli are looked at longer than their less complex counterparts, the magnitude of the effect typically has been small. Also, it is evident from the reports of Brown and Farah (1966) and Faw and Nunnally (1967) that complexity is not always an effective determinant of looking behavior. Those experiments report that variations in the instructions given to Ss can markedly alter the effects of complexity on looking behavior.

Results of the two aforementioned studies emphasize the need, expressed by Cantor et al (1963), for a systematic examination and description of the conditions under which various stimulus characteristics do and do not affect looking behavior. The present investigation is based on the hypothesis that, with relatively nonmeaningful stimuli, stimulus uncertainty is effective in directing looking behavior only to the extent that Ss are motivated to identify, attribute meaning to, remember, or in some other way encode the potential information in the stimuli. For the most part, studies of looking behavior have incorporated one or two procedures which predispose Ss to examine closely, identify, or attribute meaning to the stimuli used in the experiment. In many studies it is explained to $S$ that $E$ is concerned with the "interest value". of the stimuli, or it is suggested that the experiment concerns the length of time S "wishes" to look at the stimuli. Instructions such as these emphasize the importance of differences among the experimental stimuli and potentially encourage Ss to identify or to attribute meaning to them. Those investigators who have employed instructions which do not emphasize the importance of the stimuli typically use college sophomores as Ss (e.g., Berlyne, 1958; Faw \& Nunnally, 1967). It may be that such Ss are suspicious regarding the purposes of the experiment, and consequently they actively examine the visual display to remember or otherwise to attribute meaning to the stimuli. In contrast, it may be that children, given similar instructions, are less suspicious and less concerned with remembering or otherwise encoding the stimuli.

The purposes of the present experiment were threefold. (a) As a consequence of defining complexity in terms of physical characteristics of the stimuli, the complexity of stimuli typically employed in studies of looking behavior has been confounded by incidental physical characteristics not logically related to complexity, e.g., size, length of perimeter, and amount of contour. Recent research suggests that it is these incidental characteristics, rather than those physical characteristics logically associated with complexity, which are the effective determinants of attention in infants (McCall \& Kagan, 1967). To insure that interpretations of the present results could be based wholly on stimulus uncertainty, stimuli were employed which were equated with respect to physical characteristics not logically related to complexity but varied with respect to level of uncertainty. Furthermore, the stimuli appeared to represent a lower level of meaningfulness than has heretofore been employed. (b) The study attempted to determine if an instruction which encouraged Ss to identify and to remember stimuli, as opposed to an instruction which minimized the importance of the stimuli, would greatly enhance the effects of stimulus uncertainty on looking behavior. (c) The experiment investigated the effects of stimulus uncertainty and instructions on the looking behavior of both adults and children. Because of slight differences in the procedures employed with the two age groups, the comparison of adults and children can only provide an initial insight into potential age differences in looking behavior.

\section{Subjects}

\section{METHOD}

Subjects were 54 fourth-grade boys from schools in the Metropolitan Nashville School System and 54 adult volunteers from the married student apartments at Vanderbilt University. The ages of the children ranged from 8 to 12 years; mean age was 9.2 years. Their schools were set in what may be described as middle-class socioeconomic neighborhoods. The 25 male and 29 female adult Ss ranged in age from 19 to 39 years; mean age was 24.2 years. Eighteen Ss from each age level were assigned to one of three treatment groups.

\section{Treatment Groups}

Three treatment conditions varied the type of encouragement given Ss to encode the experimental stimuli. Ss in Group 1 engaged in a task in which they could earn money by correctly identifying stimuli as having been seen in an earlier phase of the experiment. Ss in Group 2 were pre-exposed to the experimental stimuli and given an instruction which disguised the true purpose of the experiment and minimized the importance of differences among the stimuli. To control for any effects potentially attributable to pre-exposure, Ss in Group 3 were given the same disguised-set instructions as Ss in Group 2, but they were not pre-exposed to the experimental stimuli.

\section{Apparatus}

The apparatus for recording eye movements is described in detail elsewhere (Faw \& Nunnally, 1967). It consisted of a 
viewing box with a translucent rear projection screen at one end and goggle-like opening at the other end. S looked through the opening to view the stimulus slides projected onto the screen at the far end of the box. A chin rest was used to restrict S's head movements. A timing device controlled the length of presentation of each slide. Mounted on the side of the box was a motion-picture camera which photographed the movements of S's left eye. Ear protectors were worn by $S$ to reduce auditory distractions during the viewing period.

\section{Stimuli}

The test stimuli were eight dot patterns selected from those described by Clement (1964). They were formed by placing five black dots within cells of an imaginary 3 by 3 square matrix. These stimuli have been described as representing various degrees of stimulus uncertainty (Garner \& Clement, 1963). Here the term stimulus uncertainty is used in the context proposed by Garner (1962). In that context, the uncertainty of a stimulus is considered to be a positive function of the size of the inferred subset of equivalent stimuli to which it belongs. In the case of the dot patterns described by Clement (1964), and used in the present experiment, the size of the equivalent set of stimuli has arbitrarily been defined as "the total number of different patterns which could be obtained by performing the operations of reflection (mirroring or reversal) and $90^{\circ}$ rotation, either singly or jointly [Clement, 1964 , p. 154]." Although this is an arbitrary definition of the equivalent set size, Garner and Clement (1963) report that the definition provides set sizes quite similar to those obtained by subjective sorting of the dot patterns.

Research by Clement (1964), using identical dot patterns, indicates that the verbal latency of attributing a name to a pattern, the number of distinct names attributed to it, and ratings of pattern goodness are all highly intercorrelated (e.g., all correlations were greater than .71). Furthermore, for each of these response measures the greatest percentage of variance could be explained by the independent variable of equivalent set size (i.e., pattern uncertainty). These results clearly indicate that the three subject-defined measures are operational counterparts of a single construct. Moreover, that construct can most easily be described as concerning stimulus uncertainty.

In the present study, two patterns of dots were selected to represent each of four levels of uncertainty. Here uncertainty is defined in terms of only one of the aforementioned operational counterparts of that construct-the mean latency required for adult $S$ s to attribute a name to the stimulus pattern. These latencies were obtained in Clement's original experiment (1964). The mean latencies, in seconds, for the patterns at each level are as follows: Level $1=5.19$, 5.56; Level $2=8.34,9.05$; Level $3=13.48,13.03$; Level $4=16.56$, 17.19. All possible pairs of dot patterns representing comparisons between different levels of uncertainty were photographed as stimulus slides. This resulted in two sets of 24 slides each. The sets were identical except that the left-right positions of the patterns were reversed. When projected onto the viewing screen, the patterns were separated horizontally by at least $10 \mathrm{in.,}$ and the distance between their centers subtended an arc of approximately $36 \mathrm{deg}$ as measured from the center of the goggle opening. This separation forced $S$ to make a gross eye movement in order to bring either stimulus in to his direct line of sight.

\section{Procedure}

All Ss were seen individually and familiarized with the viewing box. They were told that the experiment involved a study of the changes in the size of the pupil of the eye (the disguised-set instructions of Faw \& Nunnally, 1967). Ss were instructed to hold their heads still and to always look at some point on the viewing screen. Following these instructions, the procedures varied for the three treatment groups.

Group 1-Encouraged to identify. Following the initial instructions, each $\mathrm{S}$ was informed that he would perform a memory task as an additional part of the experiment. He was told that he would be shown a series of dot patterns on the viewing screen and that he should pay careful attention to each one, so he would be able to earn money by correctly identifying the patterns when they were shown again. Adult Ss were then shown a series of 30 dot patterns. To reduce the probability that the younger Ss might perceive this memory task as impossible, the children were shown only $20 \mathrm{dot}$ patterns. Each pattern was projected individually onto the center of the viewing screen for $5 \mathrm{sec}$. For all Ss the series included the eight test stimuli subsequently used to examine the influence of stimulus complexity on visual selection.

Following the presentation of the single patterns, $S$ was told that he would see some of those same dot patterns again, but that they would be paired on the screen. He was informed that, although almost all the patterns would be ones he had previously seen, two of the pairs would include a pattern different from any previously shown. $S$ was instructed to look for the two new dot patterns and to signal $E$ by pressing one of two buttons, placed near S's right hand, when he was sure he saw a new pattern. (One button indicated the new pattern was on the left side of the screen, while the second button indicated that the pattern was on the right.)

The instructions for the adults continued by stating that $S$ would begin the experiment with a dollar and would earn a dollar for each correct identification-a potential gain of $\$ 3.00$. However, it was emphasized that $15 ₫$ would be deducted each time $S$ made an incorrect identification. Similarly, the children were told that they would begin the experiment with $25 \$$ and would earn $10 \$$ for each correct identification and lose $3 ₫$ for each incorrect identification. It was emphasized to $S$ that he should try to correctly identify the stimuli and try not to lose any money by making errors. The differential pay rate of adults and children was based on earlier experience with children which indicated that they eagerly performed tasks for small sums of money. It was felt, however, that adults would require somewhat more incentive to participate in the task.

Group 2-Disguised instructions with pre-exposure. Ss in Group 2 were told that $\mathrm{E}$ was only interested in recording size changes in the pupil. They were pre-exposed to the individual dot-pattern slides as were the Ss in Group 1; however, Group 2 Ss were told that the pre-exposure was simply an attempt to familiarize them with all aspects of the experiment and that they would not be required to remember anything about the patterns. Following the pre-exposure period and a rest break of approximately $45 \mathrm{sec}, \mathrm{S}$ was shown the series of paired stimuli. It was emphasized to $S$ that the stimuli would merely give him something to look at during the experiment, but that what he looked at was of no importance.

Group 3-Disguised instructions without pre-exposure. The procedure for Ss in Group 3 was identical to that for Ss in Group 2 except that Ss were not exposed to the series of individual dot patterns prior to being shown the paired stimuli.

Each adult in Groups 2 and 3 was paid $\$ 2.00$, and each child in these two groups was paid $25 \nsubseteq$ for participating in the experiment.

Subjects in all groups were shown the same series of 26 stimulus slides, i.e., the 24 test slides, each comparing two levels of uncertainty and two additional slides containing one of the pre-exposure dot patterns and one novel pattern. Each slide was presented for $10 \mathrm{sec}$ and was preceded for $2 \mathrm{sec}$ by a fixation slide consisting of a single asterisk located in the 
center of the screen. The fixation slides were used to bring the S's line of vision back to the center of the screen before each stimulus slide appeared. All Ss were informed that the fixation slides would appear and were instructed to look directly at the asterisk when it was present.

To rule out bias due to position preference, the left-right positions of all test stimuli were reversed for half the Ss in each treatment group. One of eight random orders of presentation was assigned to each $\mathrm{S}$. The presentation period was divided into two parts, with a break of approximately $45 \mathrm{sec}$ between periods.

\section{RESULTS \\ Analysis of Photography}

The individual movie frames taken of each S's left eye were analyzed by an experienced judge who was uninformed about the stimuli shown to Ss. Using the corneal reflection as an index of the left eye's position, the judge recorded the eye as looking to the right, left, or center. Employing an identical method of analysis, an earlier study reported an interjudge agreement of $97.5 \%$ for a sample of 4000 frames of film (Faw \& Nunnally, 1967). For each pair of stimuli, an S's score was the percentage of time spent viewing the more uncertain stimulus. Center fixations were not used in the anlysis. Each $\mathrm{S}$ saw four stimulus slides representing comparisons between the same levels of uncertainty. For each S, scores from those four pairs were averaged to give a single score for each of the six paired comparisons among the four levels of uncertainty.

\section{Complexity and Looking Behavior}

For each of the six pair-wise comparisons between the four levels of uncertainty, separate analyses for each group of Ss examined the dominance of the more uncertain stimuli over their less uncertain counterparts. Only in groups of Ss encouraged to identify the stimuli were the more uncertain stimuli viewed significantly longer than their less uncertain counterparts. Furthermore, $t$ tests for those groups showed the mean percentages to significantly exceed the expected value of $50 \%$ only for the three comparisons in which the least uncertain stimuli was pitted against stimuli representing one of the three higher levels of uncertainty $(p<.02)$. When stimuli representing the three higher levels of uncertainty were compared with one another, more uncertain stimuli did not significantly dominate less uncertain stimuli. Thus, for groups of Ss where stimulus uncertainty was effective in influencing looking behavior the influence of uncertainty apparently reached an asymptotic level at moderate levels of uncertainty.

\section{Instructions and Age}

For Ss within the same instruction group, similar mean percentage scores were obtained for all three comparisons involving the lowest level of uncertainty. Consequently, those three percentage scores were averaged for each $S$ to produce a single score (Uncertainty Comparison 1). Similarly, the percentage scores for the three comparisons among stimuli representing the three higher levels of uncertainty did not differ for Ss within the same instruction group; therefore, those three percentage scores were averaged for each $S$ to represent Uncertainty Comparison 2. Those scores were analyzed in a 2 (age) by 3 (instruction) by 2 (uncertainty comparison) analysis of variance. The first and second factors were between-Ss factors. The third factor was a within-Ss factor represented by two levels: (1) comparisons involving the least uncertain stimuli when pitted against stimuli of higher uncertainty; (2) comparisons involving only the three higher levels of uncertainty pitted against each other. No significant differences were found between the looking behavior of adults and children, nor were any of the interactions involving the age factor significant. A significant main effect for

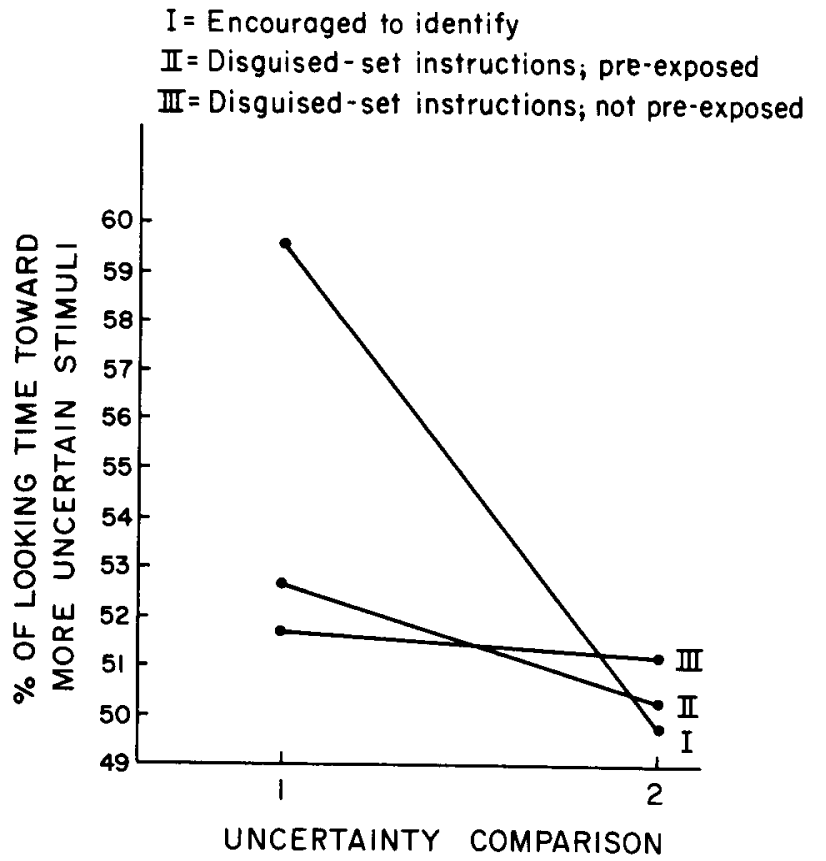

Fig. 1. Percentages of looking time to more uncertain stimuli. (Comparison 1 represents the least uncertain level compared with a more uncertain level, and Comparison 2 represents the three higher levels of uncertainty compared with one another.)

comparisons, $\quad \mathrm{F}(1,102)=25.624, \quad \mathrm{p}<.00005$, must be examined in the context of the significant interaction of that factor with the instruction factor: $F(2,102)=11.979$, $\mathrm{p}<.00005$. That interaction is pictured in Fig. 1. Analyses of the simple main effects within the Instruction by Comparison interaction, and subsequent Newman-Keuls tests, revealed that the effect of instructions was significant only for those comparisons involving stimuli representing the lowest level of uncertainty: $F(2,102)=16.50, p<.01$. Under that condition, the group instructed to identify the stimuli differed significantly from the two disguised instruction groups $(p<.01)$, while the results from the latter two groups did not differ significantly from one another.

Individual t tests of the six means revealed that only under the condition in which Ss were instructed to identify the stimuli, and then only when the comparison involved stimuli representing the lowest level of uncertainty, did the mean percentage of time spent viewing the more uncertain stimulus in the pair significantly exceed the value of $50 \%$ expected by chance $(p<.01)$.

\section{DISCUSSION}

The dominance of all stimuli over those representing the lowest level of uncertainty and the lack of dominance among the stimuli of moderate and high uncertainty is analogous to earlier reports which indicate that, under those conditions where stimulus complexity is an effective determinant of looking behavior, the relationship between complexity and looking time is positive and monotonic. Those results suggest further that the influence of uncertainty on looking behavior reaches an asymptotic level at only moderate levels of uncertainty. However, this latter result may be due in part to an artifact of the procedures. In the present experiment, stimuli were presented in pairs for a duration of 10 sec. It is plausible that when stimuli of moderate or high levels of uncertainty were compared, neither stimulus was fully identified during the 10-sec period: thus. no differential looking behavior would be expected. With increased exposure 
to those stimuli, one might observe greater differentiation in the time spent viewing the more uncertain stimulus in pairs of stimuli representing moderate and high levels of uncertainty.

The lack of significant effects due to the age of Ss or interactions of age with other factors negates the necessity for interpreting any potential influences attributable to the slightly different procedures used with the adults and children. The null results may suggest that differences in the looking behavior of adults and children are evidenced only when the experimental stimuli represent high levels of uncertainty. The present experimental procedures may partially account for the inability to observe differential looking behavior with these higher levels of uncertainty. An equally plausible explanation, however, is that with the type of stimuli and levels of uncertainty employed in the present experiment no differences exist between the looking behavior of adults and children. Further research is required to clarify the question of age differences in looking behavior.

The finding that stimulus uncertainty was influential in determining looking behavior only when Ss were encouraged to identify the stimuli clearly indicates the importance of considering uncertainty as an effective determinant of looking behavior only in the context of the task engaged in by $S$. In contrast to the present study, previous experiments concerned with looking behavior have not attempted to specify the task engaged in by $\mathrm{S}$. It has most often been assumed that Ss sat passively in the laboratory or engaged in some task unrelated to looking behavior. The present results suggest instead that Ss whose behavior is influenced by stimulus uncertainty are actively engaged in some process of stimulus identification or coding. The uncertainty of stimuli simply alters the difficulty of encoding them, the time required to encode them, and therefore the amount of time each stimulus is viewed. In experiments which employ more meaningful stimuli than those used in the present study, and in those which manipulate informational characteristics other than uncertainty (e.g., novelty or incongruity), encouragement to identify or otherwise encode the information present in the visual display may be intrinsic rather than a product of an experimental instruction; however, the differential looking behavior reported in those studies might logically be attributed to the same motivation to encode.

The results of this experiment obviously represent only one step in understanding the effects of stimulus uncertainty on looking behavior. Subsequent research should examine more closely the functional relationship between stimulus uncertainty as well as various other informational characteristics, and looking time using methods which overcome the difficulties inherent in the short-exposure, paired-comparison procedure.

More important, however, would be attempts to determine the basic processes which underlie the effect of stimulus uncertainty and other informational characteristics on looking behavior. Berlyne (1960) posits that informational characteristics are effective determinants of looking behavior in that they increase the physiological arousal of the $S$. The empirical support for this position is not conclusive. Perhaps an interpretation of looking behavior based wholly on the cognitive activity of the $S$ would prove more adequate. Such an interpretation would correspond to the position espoused by Kagan (Kagan, Henker, Hen-Tov, Levine, \& Lewis, 1966) with respect to the visual attention of infants and would fit with Gibson's theory of perceptual activity (Gibson, in press). Regardless of the final judgment, however, an adequate understanding of the variables which influence looking behavior potentially would provide important insights in to the organization of behavior.

\section{REFERENCES}

BERLYNE, D. C. The influence of complexity and novelty in visual figures on orienting responses. Journal of Experimental Psychology, 1958, 55, 289-296.

BERLYNE, D. C. Conflict, arousal, and curiosity. New York: McGraw-Hill, 1960.

BROWN, L. T., \& FARAH, W. Some physical determinants of viewing time under three instructional sets. Perception \& Psychophysics, 1966, $1,2-4$

CANTOR, G. N., CANTOR, J. H., \& DITRICHS, R. Observing behavior in preschool children as a function of stimulus complexity. Child Development, 1963, 34,683-689.

CLEMENT, D. C. Uncertainty and latency of verbal naming responses as correlates of pattern goodness. Journal of Verbal Learning \& Verbal Behavior, 1964, 3, 150-157.

FAW, T. T., \& NUNNALLY, J. C. The effects on eye movements of complexity, novelty, and affective tone. Perception \& Psychophysics,. $1967,2,263-267$.

FAW, T. T., \& NUNNALLY, J. C. The influence of stimulus complexity, novelty, and affective value on children's visual fixations. Joumal of Experimental Child Psychology, 1968, 6, 141-153.

GARNER, W. R. Uncertainty and structure in psychological concepts. New York: Wiley, 1962.

GARNER, W. R., \& CLEMENT, D. E. Goodness of pattern and pattern uncertainty. Journal of Verbal Learning \& Verbal Behavior, 1963, 2, 446452.

GIBSON, E. Principles of perceptual learning and development. New York: Appleton-Century-Crofts, in press.

KAGAN, J., HENKER, B. S., HEN-TOV, A., LEVINE, J., \& LEWIS, M. Infants' differential reactions to familiar and distorted faces. Child Development, 1966, 37, 519-532.

LECKART, B. T., \& BAKAN, P. Complexity judgments of photographs and looking time Perceptual \& Motor Skills, 1965, 2, 16-18.

McCALL, R. B., \& KAGAN, J. Attention in the infant: Effects of complexity, contour, perimeter and familiarity. Child Development, $1967,38,939-952$.

THOMAS, H. Preferences for random shapes: Ages six through nineteen years. Child Development, 1966, 37, 843-859.

\section{NOTES}

1. While this research was in progress, the first author was receiving support from a National Defense Graduate Fellowship. The study was supported, in part, by a research grant from the National Institute of Child Health and Human Development, No. HD-03083, to J.C. Nunnally. We wish to express grateful appreciation to the Metropolitan School System of Nashville, Tennessee, and to the principals and teachers of Brick Church and Glenview elementary schools for their cooperation in making Ss available for the research.

2. Address: Department of Psychology, Vanderbilt University, Nashville, Tennessee 37203.

(Accepted for publication September 23, 1968.) 